\title{
High Level View of Cloud Security: Issues AND Solutions
}

\author{
Venkata Narasimha Inukollu ${ }^{1}$, Sailaja $\mathrm{Arsi}^{1}$ and Srinivasa Rao Ravuri ${ }^{3}$ \\ ${ }^{1}$ Department of Computer Engineering, Texas Tech University, USA \\ \{narasimha.inukollu, sailaja.arsi\}@ttu.edu \\ ${ }^{3}$ Department of Banking and Financial Services, Cognizant Technology \\ Solutions, India \\ srinivasarao.ravuri@cognizant.com
}

\begin{abstract}
In this paper, we discuss security issues for cloud computing, Map Reduce and Hadoop environment. We also discuss various possible solutions for the issues in cloud computing security and Hadoop. Today, Cloud computing security is developing at a rapid pace which includes computer security, network security and information security. Cloud computing plays a very vital role in protecting data, applications and the related infrastructure with the help of policies, technologies and controls.
\end{abstract}

\section{KEYWORDS}

Cloud Computing, Big Data, Hadoop, Map Reduce, HDFS (Hadoop Distributed File System)

\section{INTRODUCTION}

In order to analyze complex data and to identify patterns it is very important to securely store, manage and share large amounts of complex data. Cloud comes with an explicit security challenge, i.e. the data owner might not have any control of where the data is placed. The reason behind this control issue is that if one wants to get the benefits of cloud computing, he/she must also utilize the allocation of resources and also the scheduling given by the controls. Hence it is required to protect the data in the midst of untrustworthy processes. Since cloud involves extensive complexity, we believe that rather than providing a holistic solution to securing the cloud, it would be ideal to make noteworthy enhancements in securing the cloud that will ultimately provide us with a secure cloud.

Google has introduced MapReduce [1] framework for processing large amounts of data on commodity hardware. Apache's Hadoop distributed file system (HDFS) is evolving as a superior software component for cloud computing combined along with integrated parts such as MapReduce. Hadoop, which is an open-source implementation of Google MapReduce, including a distributed file system, provides to the application programmer the abstraction of the map and the reduce. With Hadoop it is easier for organizations to get a grip on the large volumes of data

Sundarapandian et al. (Eds) : CCSEA, EMSA, DKMP, CLOUD, SEA - 2014

pp. 51-61, 2014. (C) CS \& IT-CSCP 2014

DOI : $10.5121 /$ csit.2014.4305 
being generated each day, but at the same time can also create problems related to security, data access, monitoring, high availability and business continuity.

In this paper, we come up with some approaches in providing security. We ought a system that can scale to handle a large number of sites and also be able to process large and massive amounts of data. However, state of the art systems utilizing HDFS and MapReduce are not quite enough/sufficient because of the fact that they do not provide required security measures to protect sensitive data. Moreover, Hadoop framework is used to solve problems and manage data conveniently by using different techniques such as combining the k-means with data mining technology [3].

\subsection{Cloud Computing}

Cloud Computing is a technology which depends on sharing of computing resources than having local servers or personal devices to handle the applications. In Cloud Computing, the word "Cloud" means "The Internet", so Cloud Computing means a type of computing in which services are delivered through the Internet. The goal of Cloud Computing is to make use of increasing computing power to execute millions of instructions per second. Cloud Computing uses networks of a large group of servers with specialized connections to distribute data processing among the servers. Instead of installing a software suite for each computer, this technology requires to install a single software in each computer that allows users to log into a Web-based service and which also hosts all the programs required by the user. There's a significant workload shift, in a cloud computing system.

Local computers no longer have to take the entire burden when it comes to running applications. Cloud computing technology is being used to minimize the usage cost of computing resources [4]. The cloud network, consisting of a network of computers, handles the load instead. The cost of software and hardware on the user end decreases. The only thing that must be done at the user's end is to run the cloud interface software to connect to the cloud. Cloud Computing consists of a front end and back end. The front end includes the user's computer and software required to access the cloud network. Back end consists of various computers, servers and database systems that create the cloud. The user can access applications in the cloud network from anywhere by connecting to the cloud using the Internet. Some of the real time applications which use Cloud Computing are Gmail, Google Calendar, Google Docs and Dropbox etc.,

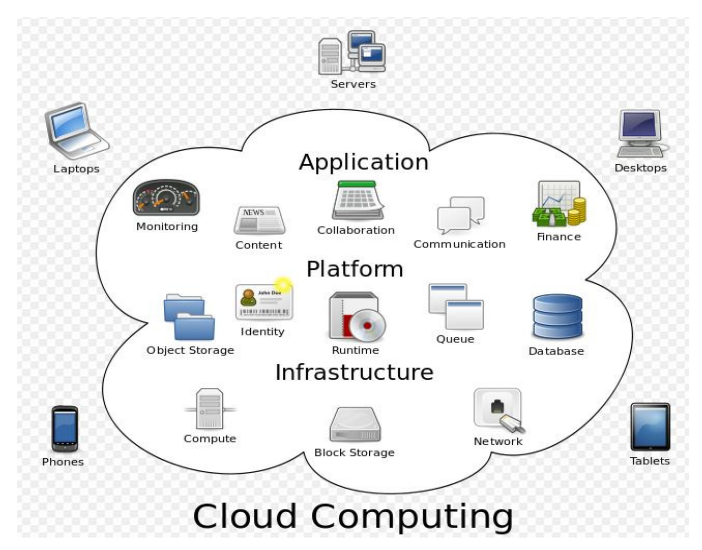

Fig1. Cloud Computing 


\subsection{Big Data}

Big Data is the word used to describe massive volumes of structured and unstructured data that are so large that it is very difficult to process this data using traditional databases and software technologies. The term "Big Data [5]" is believed to be originated from the Web search companies who had to query loosely structured very large distributed data. The three main terms that signify Big Data have the following properties:

a) Volume: Many factors contribute towards increasing Volume - storing transaction data, live streaming data and data collected from sensors etc.,

b) Variety: Today data comes in all types of formats - from traditional databases, text documents, emails, video, audio, transactions etc.,

c) Velocity: This means how fast the data is being produced and how fast the data needs to be processed to meet the demand.

The other two dimensions that need to consider with respect to Big Data are Variability and Complexity [5].

d) Variability: Along with the Velocity, the data flows can be highly inconsistent with periodic peaks.

e) Complexity: Complexity of the data also needs to be considered when the data is coming from multiple sources. The data must be linked, matched, cleansed and transformed into required formats before actual processing.

Technologies today not only support the collection of large amounts of data but also help in utilizing such data effectively. Some of the real time examples of Big Data are Credit card transactions made all over the world with respect to a Bank, Walmart customer transactions, and Facebook users generating social interaction data.

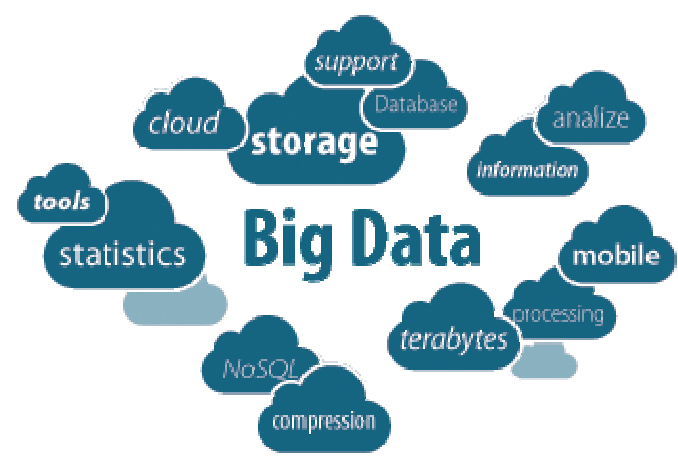

Fig2. Big Data

When making an attempt to understand the concept of Big Data, the words such as "Map Reduce" and "Hadoop" cannot be avoided. 


\subsection{Hadoop}

Hadoop, which is a free, Java-based programming framework supports the processing of large sets of data in a distributed computing environment. It is a part of the Apache project sponsored by the Apache Software Foundation. Hadoop cluster uses a Master/Slave structure [6]. Using Hadoop, large data sets can be processed across a cluster of servers and applications can be run on systems with thousands of nodes involving thousands of terabytes. Distributed file system in Hadoop helps in rapid data transfer rates and allows the system to continue its normal operation even in the case of some node failures. This approach lowers the risk of an entire system failure, even in the case of a significant number of node failures. Hadoop enables a computing solution that is scalable, cost effective, flexible and fault tolerant. Hadoop Framework is used by popular companies like Google, Yahoo, Amazon and IBM etc., to support their applications involving huge amounts of data. Hadoop has two main sub projects - Map Reduce and Hadoop Distributed File System (HDFS).

\subsection{Map Reduce}

Hadoop Map Reduce is a framework [7] used to write applications that process large amounts of data in parallel on clusters of commodity hardware resources in a reliable, fault-tolerant manner. A Map Reduce job first divides the data into individual chunks which are processed by Map jobs in parallel. The outputs of the maps sorted by the framework are then input to the reduce tasks. Generally the input and the output of the job are both stored in a file-system. Scheduling, Monitoring and re-executing failed tasks are taken care by the framework.

\subsection{Hadoop Distributed File System (HDFS)}

HDFS [8] is a file system that spans all the nodes in a Hadoop cluster for data storage. It links together file systems on local nodes to make it into one large file system. HDFS improves reliability by replicating data across multiple sources to overcome node failures.

\section{Motivation and Related Work}

\subsection{Motivation}

Along with the increasing popularity of the Could Computing environments, the security issues introduced through adaptation of this technology are also increasing. Though Cloud Computing offers many benefits, it is vulnerable to attacks. Attackers are consistently trying to find loopholes to attack the cloud computing environment. The traditional security mechanisms which are used are reconsidered because of these cloud computing deployments. Ability to visualize, control and inspect the network links and ports is required to ensure security. Hence there is a need to invest in understanding the challenges, loop holes and components prone to attacks with respect to cloud computing, and come up with a platform and infrastructure which is less vulnerable to attacks.

\subsection{Related Work}

Hadoop (a cloud computing framework), a Java based distributed system, is a new framework in the market. Since Hadoop is new and still being developed to add more features, there are many security issues which need to be addressed. Researchers have identified some of the issues and started working on this. Some of the notable outcomes, which is related to our domain and helped us to explore, are presented below. 
The World Wide Web consortium has identified the importance of SPARQL which can be used in diverse data sources. Later on, the idea of secured query was proposed in order to increase privacy in privacy/utility tradeoff. Here, Jelena, of the USC Information Science Institute, has explained that the queries can be processed according to the policy of the provider, rather than all query processing. Bertino et al published a paper on access control for XML Documents [9]. In the paper, cryptography and digital signature technique are explained, and techniques of access control to XML data document is stressed for secured environment. Later on, he published another paper on authentic third party XML document distribution [10] which imposed another trusted layer of security to the paradigm. Moreover, Kevin Hamlen and et al proposed that data can be stored in a database encrypted rather than plain text. The advantage of storing data encrypted is that even though intruder can get into the database, he or she can't get the actual data. But, the disadvantage is that encryption requires a lot of overhead. Instead of processing the plain text, most of the operation will take place in cryptographic form. Hence the approach of processing in cryptographic form added extra to security layer.

IBM researchers also explained that the query processing should take place in a secured environment. Then, the use of Kerberos has been highly effective. Kerberos is nothing but a system of authentication that has been developed at MIT. Kerberos uses an encryption technology along with a trusted third party, an arbitrator, to be able to perform a secure authentication on an open network. To be more specific, Kerberos uses cryptographic tickets to avoid transmitting plain text passwords over the wire. Kerberos is based upon Needham-Schroeder protocol. Airavat [11] has shown us some significant advancement security in the Map Reduce environment. In the paper, Roy and et al have used the access control mechanism along with differential privacy. They have worked upon mathematical bound potential privacy violation which prevents information leak beyond data provider's policy.

The above works have influenced us, and we are analyzing various approaches to make the cloud environment more secure for data transfer and computation.

\section{ISSUES AND CHALLENGES}

Cloud computing comes with numerous security issues because it encompasses many technologies including networks, databases, operating systems, virtualization, resource scheduling, transaction management, load balancing, concurrency control and memory management. Hence, security issues of these systems and technologies are applicable to cloud computing. For example, it is very important for the network which interconnects the systems in a cloud to be secure. Also, virtualization paradigm in cloud computing results in several security concerns. For example, mapping of the virtual machines to the physical machines has to be performed very securely. Data security not only involves the encryption of the data, but also ensures that appropriate policies are enforced for data sharing. In addition, resource allocation and memory management algorithms also have to be secure. Finally, data mining techniques may also be used in the malware detection in clouds.

The challenges of security in cloud computing environments are discussed below.

\subsection{Distributed Nodes}

Distributed nodes [12] are an architectural issue. The computation is done in any set of nodes. Basically, data is processed in those nodes which have the necessary resources. Since it can 
happen anywhere across the clusters, it is very difficult to find the exact location of computation. Because of this it is very difficult to ensure the security of the place where computation is done.

\subsection{Distributed Data}

In order to alleviate parallel computation, a large data set can be stored in many pieces across many machines. Also, redundant copies of data are made to ensure data reliability. In case a particular chunk is corrupted, the data can be retrieved from its copies. In the cloud environment, it is extremely difficult to find exactly where pieces of a file are stored. Also, these pieces of data are copied to another node/machines based on availability and maintenance operations. In traditional centralized data security system, critical data is wrapped around various security tools. This cannot be applied to cloud environments since all related data are not presented in one place and it changes.

\subsection{Internode Communication}

Much Hadoop distributions use RPC over TCP/IP for user data/operational data transfer between nodes. This happens over a network, distributed around globe consisting of wireless and wired networks. Therefore, anyone can tap and modify the inter node communication[12] for breaking into systems.

\subsection{Data Protection}

Many cloud environments like Hadoop store the data as it is without encryption to improve efficiency. If a hacker can access a set of machines, there is no way to stop him to steal the critical data present in those machines.

\subsection{Administrative Rights for Nodes}

A node has administrative rights [12] and can access any data. This uncontrolled access to any data is very dangerous as a malicious node can steal or manipulate critical user data.

\subsection{Authentication of Applications and Nodes}

Nodes can join clusters to increase the parallel operations. In case of no authentication, third part nodes can join clusters to steal user data or disrupt the operations of the cluster.

\subsection{Logging}

In the absence of logging in a cloud environment, no activity is recorded which modify or delete user data. No information is stored like which nodes have joined cluster, which Map Reduce jobs have run, what changes are made because of these jobs. In the absence of these logs, it is very difficult to find if someone has breached the cluster if any, malicious altering of data is done which needs to be reverted. Also, in the absence of logs, internal users can do malicious data manipulations without getting caught.

\subsection{Traditional Security Tools}

Traditional security tools are designed for traditional systems where scalability is not huge as cloud environment. Because of this, traditional security tools which are developed over years 
cannot be directly applied to this distributed form of cloud computing and these tools do not scale as well as the cloud scales.

\subsection{Use of Different Technologies}

Cloud consists of various technologies which has many interacting complex components. Components include database, computing power, network, and many other stuff. Because of the wide use of technologies, a small security weakness in one component can bring down the whole system. Because of this diversity, maintaining security in the cloud is very challenging.

\section{The Proposed APPROACHES}

We present various security measures which would improve the security of cloud computing environment. Since the cloud environment is a mixture of many different technologies, we propose various solutions which collectively will make the environment secure. The proposed solutions encourage the use of multiple technologies/ tools to mitigate the security problem specified in previous sections. Security recommendations are designed such that they do not decrease the efficiency and scaling of cloud systems.

Following security measures should be taken to ensure the security in a cloud environment.

\subsection{File Encryption}

Since the data is present in the machines in a cluster, a hacker can steal all the critical information. Therefore, all the data stored should be encrypted. Different encryption keys should be used on different machines and the key information should be stored centrally behind strong firewalls. This way, even if a hacker is able to get the data, he cannot extract meaningful information from it and misuse it. User data will be stored securely in an encrypted manner.

\subsection{Network Encryption}

All the network communication should be encrypted as per industry standards. The RPC procedure calls which take place should happen over SSL so that even if a hacker can tap into network communication packets, he cannot extract useful information or manipulate packets.

\subsection{Logging}

All the map reduce jobs which modify the data should be logged. Also, the information of users, which are responsible for those jobs should be logged. These logs should be audited regularly to find if any, malicious operations are performed or any malicious user is manipulating the data in the nodes.

\subsection{Software Format and Node Maintenance}

Nodes which run the software should be formatted regularly to eliminate any virus present. All the application softwares and Hadoop software should be updated to make the system more secure. 


\subsection{Nodes Authentication}

Whenever a node joins a cluster, it should be authenticated. In case of a malicious node, it should not be allowed to join the cluster. Authentication techniques like Kerberos can be used to validate the authorized nodes from malicious ones.

\subsection{Rigorous System Testing of Map Reduce Jobs}

After a developer writes a map reduce job, it should be thoroughly tested in a distributed environment instead of a single machine to ensure the robustness and stability of the job.

\subsection{Honeypot Nodes}

Honey pot nodes should be present in the cluster, which appear like a regular node but is a trap. These honeypots trap the hackers and necessary actions would be taken to eliminate hackers.

\subsection{Layered Framework for Assuring Cloud}

A layered framework for assuring cloud computing [13] as shown in Figure (1) consists of the secure virtual machine layer, secure cloud storage layer, secure cloud data layer, and the secure virtual network monitor layer. Cross cutting services are rendered by the policy layer, the cloud monitoring layer, the reliability layer and the risk analysis layer.

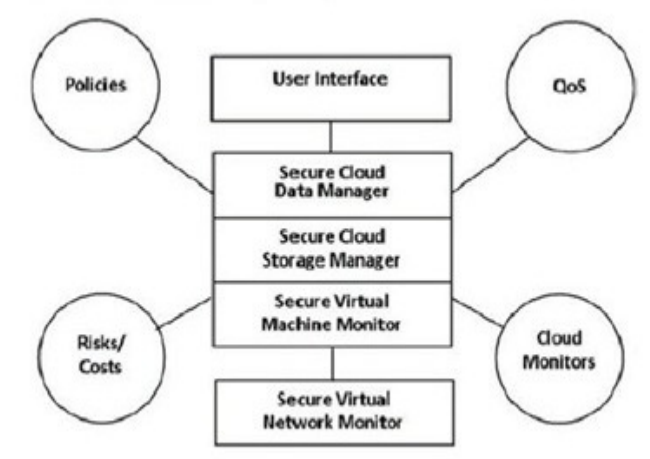

Fig3: Layered framework for assuring cloud [13]

\subsection{Third Party Secure Data Publication to Cloud}

Cloud computing helps in storing of data at a remote site in order to maximize resource utilization. Therefore, it is very important for this data to be protected and access should be given only to authorized individuals. Hence this fundamentally amounts to secure third party publication of data that is required for data outsourcing, as well as for external publications. In the cloud environment, the machine serves the role of a third party publisher, which stores the sensitive data in the cloud. This data needs to be protected, and the above discussed techniques have to be applied to ensure the maintenance of authenticity and completeness. 


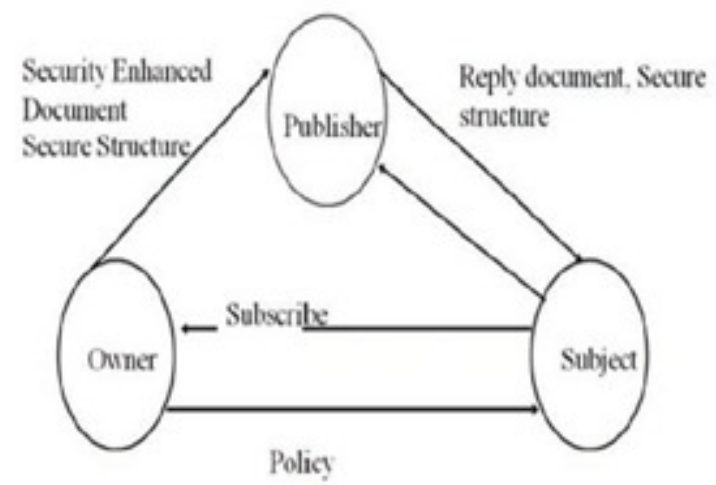

Fig4: Third party secure data publication applied to cloud [13].

\subsection{Access Control}

Integration of mandatory access control and differential privacy in distributed environment will be a good security measure. Data providers will control the security policy of their sensitive data. They will also control the mathematical bound on privacy violation that could take place. In the above approach, users can perform data computation without any leakage of data. To prevent information leak, SELinux [14] will be used. SELinux is nothing but Security-Enhanced Linux, which is a feature that provides the mechanism for supporting access control security policy through the use of Linux Security Modules (LSM) in the Linux Kernel. Enforcement of differential privacy will be done using modification to Java Virtual Machine and the Map Reduce framework. It will have inbuilt applications which store the user identity pool for the whole cloud service. So the cloud service will not have to maintain each user's identity for each application. In addition to the above methodologies, cloud service will support third party authentication. The third party will be trusted by both the cloud service and accessing user. Third party authentication will add an additional security layer to the cloud service.

Real time access control will be a good security measure in the cloud environment. In addition to access control to the cloud environment, operational control within a database in the cloud can be used to prevent configuration drift and unauthorized application changes. Multiple factors such as IP address, time of the day, and authentication method can be used in a flexible way to employ above measures. For example, access can be restricted to specific middle tier, creating a trusted path to the data. Keeping a security administrator separate from the database administrator will be a good idea. The label security method will be implemented to protect sensitive data by assigning data label or classifying data.

Data can be classified as public, confidential and sensitive. If the user label matches with the label of the data, then access is provided to the user. Examination of numerous data breaches has shown that auditing could have helped in early detection of problems and avoids them. Auditing of events and tracking of logs taking place in the cloud environment will enable possible attack. Fine grain auditing just like Oracle 9i enables conditional auditing on the specific application column. 


\section{ConClusion}

Cloud environment is widely used in industry; therefore security is an important aspect for businesses running on these cloud environments. Using proposed approaches, cloud environments can be secured for complex business operations.

\section{REFERENCES}

[1] Ren, Yulong, and Wen Tang. "A SERVICE INTEGRITY ASSURANCE FRAMEWORK FOR CLOUD COMPUTING BASED ON MAPREDUCE."Proceedings of IEEE CCIS2012. Hangzhou: 2012, pp 240 - 244, Oct. 30 2012-Nov. 12012

[2] N, Gonzalez, Miers C, Redigolo F, Carvalho T, Simplicio M, de Sousa G.T, and Pourzandi M. "A Quantitative Analysis of Current Security Concerns and Solutions for Cloud Computing.". Athens: 2011., pp 231 - 238, Nov. 29 2011- Dec. 12011

[3] Hao, Chen, and Ying Qiao. "Research of Cloud Computing based on the Hadoop platform.". Chengdu, China: 2011, pp. 181 - 184, 21-23 Oct 2011.

[4] Y, Amanatullah, Ipung H.P., Juliandri A, and Lim C. "Toward cloud computing reference architecture: Cloud service management perspective.". Jakarta: 2013, pp. 1-4, 13-14 Jun. 2013.

[5] A, Katal, Wazid M, and Goudar R.H. "Big data: Issues, challenges, tools and Good practices.". Noida: 2013, pp. 404 - 409, 8-10 Aug. 2013.

[6] Lu, Huang, Ting-tin Hu, and Hai-shan Chen. "Research on Hadoop Cloud Computing Model and its Applications.". Hangzhou, China: 2012, pp. 59 - 63, 21-24 Oct. 2012.

[7] Wie, Jiang, Ravi V.T, and Agrawal G. "A Map-Reduce System with an Alternate API for Multi-core Environments.". Melbourne, VIC: 2010, pp. 84-93, 17-20 May. 2010.

[8] K, Chitharanjan, and Kala Karun A. "A review on hadoop - HDFS infrastructure extensions.". JeJu Island: 2013, pp. 132-137, 11-12 Apr. 2013.

[9] Bertino, Elisa, Silvana Castano, Elena Ferrari, and Marco Mesiti. "Specifying and enforcing access control policies for XML document sources." pp 139-151.

[10] E, Bertino, Carminati B, Ferrari E, Gupta A, and Thuraisingham B. "Selective and Authentic ThirdParty Distribution of XML Documents."2004, pp. 1263 - 1278.

[11] Kilzer, Ann, Emmett Witchel, Indrajit Roy, Vitaly Shmatikov, and Srinath T.V. Setty. "Airavat: Security and Privacy for MapReduce."

[12] "Securing Big Data: Security Recommendations for Hadoop and NoSQL Environments." Securosis blog, version 1.0 (2012)

[13] P.R , Anisha, Kishor Kumar Reddy C, Srinivasulu Reddy K, and Surender Reddy S. "Third Party Data Protection Applied To Cloud and Xacml Implementation in the Hadoop Environment With Sparql."2012. 39-46, Jul - Aug. 2012.

[14] "Security-Enhanced Linux." Security-Enhanced Linux. N.p. Web. 13 Dec 2013. 


\section{Authors}

\section{Venkata Narasimha Inukollu}

PhD Student

Department of Computer Science \& Engineering

Texas Tech University, USA.

B.E. (Information technology \& Science), 2004, VRSEC, Vijayawada, INDIA.

M.E. (Software Engineering), 2007, BITS-Pilani, Rajasthan, INDIA

Areas of Interest: Software Engineering, Mobile software engineering, secure software engineering, secure specifications.

\section{Sailaja Arsi}

MS Student

Department of Computer Science \& Engineering

Texas Tech University, USA.

\section{Srinivasa Rao Ravuri}

Project Lead

Department of Banking and Financial Services, Cognizant Technology Solutions, India

B. Tech. (Instrumentation engineering), 2004, VRSEC, Vijayawada, INDIA.

M. Tech. (Instrumentation engineering), 2006, Anna University, Chennai, INDIA. 